\title{
Die Schweizerische Gesellschaft für Musik-Medizin
}

\author{
Die Schweizerische Gesellschaft für Musik-Medizin (SMM) wurde im Jahr 1997 ins \\ Leben gerufen. Sie dient gesamtschweizerisch als Anlauf- und Beratungsstelle für \\ Musikermedizin, die als spezifischer Teil der Arbeitsmedizin zu verstehen ist. Sie \\ umfasst Ärzte, Zahnärzte und Therapeuten verschiedener Art.
}

Korrespondenz:

Dr. med. Adrian Sury FMH Allgemeinmedizin und Betriebsmedizin SGARM Via della Posta 44 CH-Locarno

adrian.sury@hin.ch

Anlauf- und Beratungsstelle für Musikermedizin Rumiweg 4 CH-4539 Farnern

info@musik-medizin.ch

www.musik-medizin.ch
Immer häufiger werden Musiker und Musikerinnen in ihrer Spielfähigkeit eingeschränkt oder sogar berufsunfähig, da sie an einem spezifischen, gesundheitlichen Problem leiden. Heute ist Profimusik mit Spitzensport vergleichbar, und es werden Höchstleistungen verlangt. Oft führen symptomatische Therapien lediglich zu einer kurzfristigen Besserung oder verschlimmern sogar die Beschwerden. Wichtig ist, dass die Ursachen erkannt und die Musiker in ihrer Spezifizität ernst genommen werden.

Und genau dafür setzt sich die Schweizerische Gesellschaft für Musik-Medizin (SMM) ein. Sie arbeitet zusammen mit ausgewählten Spezialisten und Institutionen, die mehrere Jahre Erfahrung auf dem Gebiet der Musik-Medizin haben und medizinische sowie musikalische Kenntnisse mitbringen. Sie fördert die intensive interdisziplinäre Zusammenarbeit zwischen Ärzten, Zahnärzten und Therapeuten, damit der Musiker als Ganzes erfasst werden kann.

Die SMM hat in der Schweiz in Sachen Prävention und Therapie von gesundheitlichen Störungen der Musiker Pionierarbeit geleistet. Als der Zahnarzt, Trompeter und ehemalige Vizepräsident der SMM, Dr. med. dent. Markus Keller, vor zwei Jahrzehnten bei der Verfassung seiner Dissertation «Prothetische Probleme bei der Behandlung von Blasmusikern» Fachleute oder Publikationen in Mitteleuropa oder gar in der Schweiz suchte, blieb er leider erfolglos. Damals fand man einige wenige Musikmediziner allenfalls in England oder der damaligen DDR, wo zum Beispiel im Betriebsambulatorium der Berliner Bühnen bereits eine brauchbare Arbeitsmedizin für Musiker existierte. Nicht so in unseren Gefilden, wo in dieser Hinsicht gähnende Leere herrschte. Diesem unerfreulichen Zustand hat die Kinesiologin und Berufsmusikerin (Posaunistin) Pia Bucher zusammen mit dem Zahnarzt und Pianisten Joachim Lahme 1997 mit der Gründung der SMM glücklicherweise ein Ende gesetzt. Es ist ihr grosser Verdienst, dass die Musikmedizin in der Schweiz ihren festen Platz gefunden hat. Durch viele Fachvorträge, Weiterbildungskurse, Symposien und Dokumentationen wurde die SMM zu dem gemacht, was sie heute ist: eine über unsere Landesgrenzen hinaus anerkannte und vernetzte Fachgesellschaft, Wei-

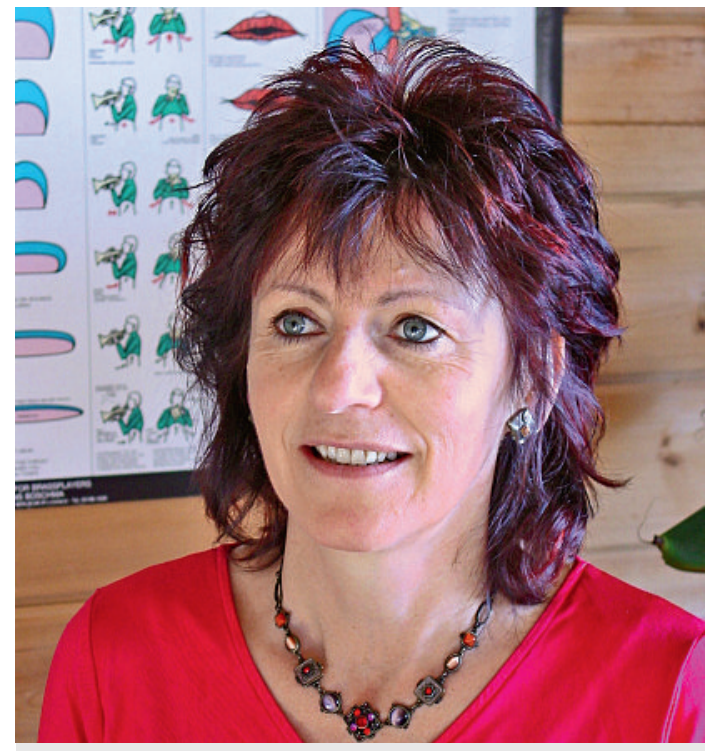

Die langjährige Präsidentin und Gründerin der Gesellschaft Pia Bucher wurde für ihre Verdienste zur Ehrenpräsidentin ernannt.

terbildungsorganisation und Anlaufstelle für Musiker, Ärzte und Therapeuten, denen das medizinische Wohl von Künstlerinnen und Künstlern, aber auch von vielen Amateurmusikern ein ernstes Anliegen ist. Die Gesellschaft zählt heute 117 Aktivmitglieder, die in Musikersprechstunden in allen Landesteilen interdisziplinär organisiert sind.

Am 22. Januar 2011 ist die langjährige Präsidentin und Gründerin der SMM Pia Bucher von ihrem Amt zurückgetreten; als Nachfolgerin wurde Martina Berchtold, Psychologin und Geigerin, gewählt. Das Vizepräsidium übernimmt der Internist und Flötist Dr. med. Jürg Frei, weitere Vorstandsmitglieder sind der Physiotherapeut und Bassist Fredy Bopp und die Pädagogin und Klarinettistin Sylvia Schwarzenbach. Ein fachlicher Beirat aus Ärzten, Zahnärzten und Therapeuten aus allen Landesteilen vervollständigt das Leitungsteam. Pia Bucher wurde für ihre Verdienste als Ehrenpräsidentin akklamiert.

Das nächste Symposium wird am 22. Oktober 2011 an der Hochschule der Künste in Bern stattfinden. 